\title{
Test Power Reduction Through Minimization of Scan Chain Transitions*
}

\author{
Ozgur Sinanoglu, Ismet Bayraktaroglu and Alex Orailoglu \\ Computer Science and Engineering Department \\ University of California, San Diego \\ La Jolla, CA 92093 \\ \{ozgur, ibayrakt, alex\}@cs.ucsd.edu
}

\begin{abstract}
Parallel test application helps reduce the otherwise considerable test times in SOCs; yet its applicability is limited by average and peak power considerations. The typical test vector loading techniques result in frequent transitions in the scan chain, which in turn reflect into significant levels of circuit switching unnecessarily. Judicious utilization of logic in the scan chain can help reduce transitions while loading the test vector needed. No performance degradation ensues as scan chain modifications have no impact on functional execution. A computationally efficient scheme is proposed to identify the location and type of the logic to be inserted. The experimental results confirm the significant reductions in test power possible under the proposed scheme.
\end{abstract}

\section{Introduction}

Testing in parallel the cores in a System-On-a-Chip (SOC) helps reduce test application time; yet power dissipation imposes strict limits to this parallelism as overheating the chip might result in damaging the cores under test. Not only does average power dissipation during testing need to be reduced to avoid overheating, but particular attention has to be devoted to peak power dissipation issues furthermore, as exceeding power thresholds may burn the chip. While decreasing test clock frequency does reduce test power, it results in significantly longer test application time. Instead, lowering switching activity, estimated as the transition count of a gate output, reduces test power, yet at no prolongation in test time.

During test mode, filling in the scan chain with the test data requires shifting the bits one by one into each chain, thus creating increased switching activity in the flip-flops. The rippling effect in a scan chain reflects into the circuit, resulting in a large number of unnecessary transitions at the circuit lines. Average test power can be reduced by preventing the unnecessary rippling in the scan chain during the shift of the test data.

\footnotetext{
${ }^{*}$ The work of the first two authors has been supported through graduate fellowships by IBM.
}

Traditionally, the data inserted to the scan chain is identical to the actual test vectors applied to the circuit under test. Modifying the scan chain through the insertion of logic between the scan cells, however, breaks this relationship; the input stimuli inserted to the scan chain may differ from the test vectors. As the number of transitions in a scan chain is strictly determined by the input stimuli inserted to the scan chain, modifications in the scan chain can be performed so as to enable the application of stimuli with fewer embedded transitions. An analysis of the original test vectors and their transition frequencies can be used to determine effectively the appropriate scan chain modifications which yield the stimuli with reduced transition cost.

We present a methodology wherein the scan chain is modified by inserting logic gates between the scan cells to reduce the number of transitions. Reduction of the switching activity during the shift-in of the test stimuli in turn results in a decrease in test power. The methodology presented herein proposes the introduction of gate delays only to the scan path; hence the functional operation of the circuit is kept intact timing-wise. The approach is quite efficient and easily implementable. It is compatible with the conventional test flow used in industry and can work with any kind of ATPG tool. The methodology is applicable as well regardless of whether the test vectors are fully or partially specified.

\section{Previous Work}

In recent years, a considerable amount of effort has been expended in reducing test power for embedded cores in a SOC; the proposed solutions in the literature have focused on reducing the switching activity in the circuit. The transitions that originate from the scan chain can be prevented from propagating into the circuit through the use of externally controlled gates [1]; however, such techniques result in performance degradation as they necessitate gate delay insertion on critical paths.

A combinational ATPG methodology, which generates test vectors with minimized power dissipation, is proposed in [2]. Transition controllability and observability cost func- 
tions are defined and used to guide the backtrace and objective selection procedures of ATPG. However, incorporating power optimizations into ATPG algorithms typically results in test application time prolongation.

In scan-based testing, the test data should be brought to the primary inputs before the apply/capture cycle; the primary inputs can therefore be set arbitrarily during the preceding shift-in cycles. In [3], a technique is presented, exploiting this observation, wherein the values, which are assigned to the primary inputs during shift operations, are computed for reducing the transitions in the circuit. Nicolici et. al. [4], exploiting the same observation, propose a scheme which determines the most appropriate shift-in cycle for updating primary inputs so as to reduce test power. The effectiveness of such techniques is checkmated somewhat, since typical circuits are controlled mostly by scan chains rather than primary inputs; scan chain transitions create rippling at the circuit lines despite primary input adjustments.

Several techniques in the literature aim at reducing test power through decreasing the number of transitions in the scan chain. In [5], test cubes are compacted and padded so as to minimize the number of scan chain transitions; even though test power is reduced, test application time might be slightly increased in comparison to the random padding case. Furthermore, the applicability of this technique is limited by the density of the unspecified bits. In [6], the scan chain is partitioned into several scan chains. Activation of only one scan chain at a time restricts rippling, reducing the test power dissipated, yet resulting in a commensurate test application time increase. To minimize the switching activity in the scan chain, two techniques, test vector ordering and scan-latch ordering, are proposed in [7]; however, the computational complexity of these techniques limits their applicability.

A distinct vein of research effort aims at incorporating test power as a constraint into test scheduling. Chou et. al. [8] for example propose a test scheduling technique wherein the power constraint is embedded in the resource allocation graph to determine the optimal scheduling of the tests under a power constraint. The increase in test application time due to the additional constraints incorporated into the scheduling problem is confirmed by the experimental results presented.

\section{Motivation}

The test set provided along with the core can be analyzed to pinpoint the indices of specific bits wherein the transition frequency is heightened; based on this analysis, the scan chain can be modified. The test stimulus to be inserted into the scan chain needs to be computed by transforming the original test vectors to reflect the effects of the modification. The scan chain modification enables the application of a new stimulus with a significantly reduced number of transitions. An example can be seen in Figure 1, where the application of "00110" to an unmodified scan chain results in rippling,

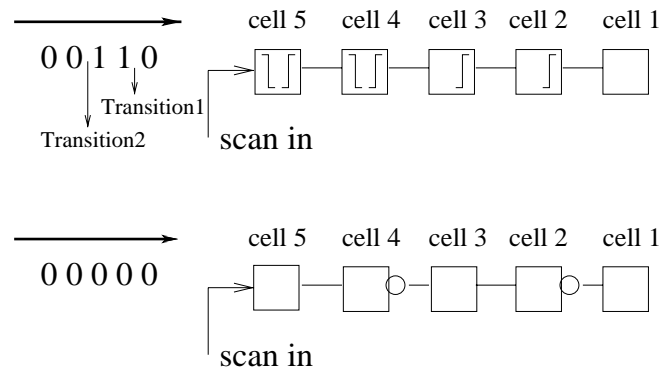

\section{Figure 1. The impact of vector transformation on the rippling of the scan cells}

yet the insertion of " 00000 " to the modified scan chain results in no rippling whatsoever, while delivering the actual test vector, "00110" to the circuit under test inputs. The rippling effect in the scan chain is eliminated, which in turn yields reduced power dissipation in both the chain and most importantly in the circuit during shifting in the test stimuli.

As large test sets typically preclude accidental dependencies in the test data, the modifications should be bijective; distinct test vectors should be mapped to distinct stimuli. As functions with controlling values such as AND and OR preclude delivery of particular test vectors to the modified scan chain, we restrict the gates under consideration to ones with no controlling value.

Even with the restriction of the possible scan chain modifications to inverter and $\mathrm{X}(\mathrm{N}) \mathrm{OR}$ insertion, still the cardinality of the candidate transformation set can be exceedingly large. For a scan chain of length $n, 4^{n}$ scan chain modifications exist; between any two consecutive scan cells, the inclusion of an XOR, an XNOR, or an inverter needs to be considered in addition to simply leaving this point unmodified. Computational efficiency necessitates independent modifications in various scan chain fragments; the optimal modification in each scan chain fragment should be individually implemented by analyzing the corresponding bits of the test vectors. Test vector data should therefore be decomposed into blocks, each containing a number of consecutive bits. Consideration of every transition necessitates a single bit overlap between any two consecutive blocks.

Independence of modifications can be guaranteed through limiting their impact to a single test data block; if the modification of a certain part of the scan chain affects solely the transitions embedded in a single test data block, each test data block can be analyzed and transformed independently. Such a local modification impact can be achieved only if the modification is transition-preserving for the test bits that pass through the modified scan chain fragment; a modification implemented in a certain part of the scan chain should impact only the transitions between the bits to be delivered into the scan cells involved in the modification.

An inverter, for example, between two scan cells necessitates transformation of the actual test data that passes through 


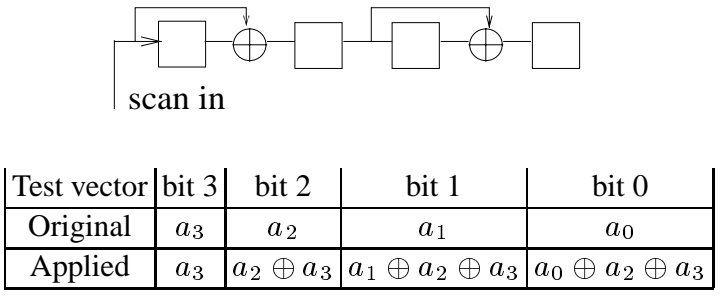

Figure 2. Impact of XOR gates on the transitions between test data bits

this gate; the applied stimulus bits which are to pass through the inverter should equal the original test data bits negated. The only transition that is affected by such a modification is the one between the two bits, only one of which is negated; all the other transitions remain intact. Therefore, this modification is indeed a transition-preserving one as it has a local impact on the transitions between the test data bits; contiguous scan chain bit pairs can thus be modified independently of the remaining bits through the insertion of inverters.

$\mathrm{X}(\mathrm{N}) \mathrm{OR}$ functions, on the other hand, have accumulative effects; a modification consisting of the insertion of a single $\mathrm{X}(\mathrm{N}) \mathrm{OR}$ gate is not transition-preserving as it fails to keep intact the transitions between the test bits that pass through this gate. Figure 2 illustrates this point for the case of an XOR gate, wherein it can be seen that all the bits except the leftmost ${ }^{1}$ ones in the applied stimulus have to be recomputed; a trivially analogous case holds for XNORs. Utilization of the $\mathrm{X}(\mathrm{N}) \mathrm{OR}$ function in a transition-preserving manner necessitates masking the effect of an $\mathrm{X}(\mathrm{N}) \mathrm{OR}$ gate with another one in the subsequent bit location, as illustrated in Figure 3, enabling the examination of test data in small chunks of three bits. The overall impact of such a modification is thus limited to two consecutive transitions either side of the middle cell. As the applied stimulus bits that are to pass through two $\mathrm{X}(\mathrm{N}) \mathrm{OR}$ gates in consecutive locations either remain intact or are negated, such a modification is a transition-preserving one. The local impact of this modification enables the utilization of $\mathrm{X}(\mathrm{N}) \mathrm{OR}$ gates together with inverters for modifying scan chain fragments.

If for the majority of the test vectors, a transition occurs between two adjacent bit locations, inserting an inverter between the corresponding two scan cells results in reducing the transition frequency in these bit locations. A transition frequency of $80 \%$, for instance, can be reduced down to $20 \%$ through such a modification. Test data distribution within a block therefore determines the effectiveness of implementing a modification that provides a specific transformation; the modification that yields the applied stimuli with the minimum number of transitions is selected for a specific scan chain fragment.

The test power reduction technique can be utilized in a

\footnotetext{
${ }^{1}$ The test vector bits with lower numbered indices are shifted into the scan chain prior to the ones with higher indices.
}

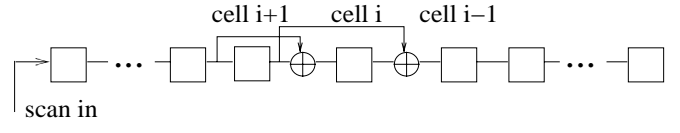

\begin{tabular}{|c|c|c|c|c|c|} 
Test vector & bit i+2 & bit i+1 & bit i & bit i-1 & bit i-2 \\
\hline Original & $a_{i+2}$ & $a_{i+1}$ & $a_{i}$ & $a_{i-1}$ & $a_{i-2}$ \\
\hline Applied & $a_{i+2}$ & $a_{i+1}$ & $a_{i} \oplus a_{i+1}$ & $a_{i-1}$ & $a_{i-2}$ \\
\hline
\end{tabular}

Figure 3. A modification implemented in the scan chain using XOR gates

similar manner to handle test responses instead, reducing power dissipation during the shift-out of test responses. An intertwined solution, wherein both test vectors and test responses are considered, imposes significant additional challenges as decisions in one domain impact the other in complex ways. We focus only on the reduction of test power dissipation during the shift-in of the test stimuli, in the rest of this work.

\section{Algorithmic Framework}

In this section the possible scan chain modifications are presented. The impact of these modifications on the transitions in the scan chain is analyzed so as to build a selection methodology for particular scan chain fragments. An algorithm for average test power reduction based on test data analysis is provided. The outcome of this algorithm consists of generating the appropriate modifications in the scan chain which enable the application of power-efficient stimuli; each stimulus block is computed based on the functionality of the modification implemented in the corresponding scan chain fragment.

\subsection{Possible Scan Chain Modifications}

Table 1 lists the transformations associated with various scan chain modifications. The second column denotes the gates to be inserted between the elements of the left pair and the gates to be inserted between the elements of the right pair, in that order. For every modification, the stimulus that needs to be applied to bring in the three actual test vector bits into the scan cells is provided. It is important to note that for all the entries in table 1 , the rightmost test bit, $a_{i-1}$, ei-

\begin{tabular}{|c|c|c|c|c|}
\hline & \multirow{2}{*}{ Gates } & \multicolumn{3}{|c|}{ Test Bit } \\
\cline { 3 - 5 } & $a_{i+1}$ & $a_{i}$ & $a_{i-1}$ \\
\hline F1 & XOR,XOR & $a_{i+1}$ & $a_{i} \oplus a_{i+1}$ & $a_{i-1}$ \\
\hline F2 & XNOR,XNOR & $a_{i+1}$ & $\left(a_{i} \oplus a_{i+1}\right)^{\prime}$ & $a_{i-1}$ \\
\hline F3 & INV,INV & $a_{i+1}$ & $a_{i}^{\prime}$ & $a_{i-1}$ \\
\hline F4 & XOR,XNOR & $a_{i+1}$ & $a_{i} \oplus a_{i+1}$ & $a_{i-1}^{\prime}$ \\
\hline F5 & XNOR,XOR & $a_{i+1}$ & $\left(a_{i} \oplus a_{i+1}\right)^{\prime}$ & $a_{i-1}^{\prime}$ \\
\hline F6 & INV,- & $a_{i+1}$ & $a_{i}^{\prime}$ & $a_{i-1}^{\prime}$ \\
\hline F7 &,- INV & $a_{i+1}$ & $a_{i}$ & $a_{i-1}^{\prime}$ \\
\hline F8 &,-- & $a_{i+1}$ & $a_{i}$ & $a_{i-1}$ \\
\hline
\end{tabular}

Table 1. Scan chain modifications 


\begin{tabular}{|c|c|c|c|c|c|c|c|c|}
\hline$a_{i+1} a_{i} a_{i-1}$ & F1 & F2 & F3 & F4 & F5 & F6 & F7 & F8 \\
\hline 000 & 00 & -- & -- & $0-$ & -0 & -0 & $0-$ & 00 \\
\hline 001 & 00 & -+ & -+ & $0+$ & -0 & -0 & $0+$ & 00 \\
\hline 010 & 00 & ++ & ++ & $0+$ & +0 & +0 & $0+$ & 00 \\
\hline 011 & 00 & +- & +- & $0-$ & +0 & +0 & $0-$ & 00 \\
\hline 100 & +- & 00 & +- & +0 & $0-$ & +0 & $0-$ & 00 \\
\hline 101 & ++ & 00 & ++ & +0 & $0+$ & +0 & $0+$ & 00 \\
\hline 110 & -+ & 00 & -+ & -0 & $0+$ & -0 & $0+$ & 00 \\
\hline 111 & -- & 00 & -- & -0 & $0-$ & -0 & $0-$ & 00 \\
\hline
\end{tabular}

Table 2. Impact of the modifications on the transitions between test data bits

ther remains intact or is negated, thus ensuring the transitionpreserving property for all such modifications.

The distinct local impact of every modification on the transitions within a block of test data is demonstrated in table 2; for all eight combinations of three test data bits, the effect of implementing a modification on the transitions between the first two and the last two bit positions in the block, respectively, is provided. A transition that is removed is denoted by "+", a transition created due to the modification of the scan chain, by "-"; "0" denotes no impact. For instance, inserting 2 XOR gates results in removing both transitions when the test data bits are "101", since " 111 " should be inserted to the scan chain to apply "101" to the circuit under test.

As implementing these transition-preserving modifications enables independent modifications in the scan chain, the test data can be decomposed into blocks that can be analyzed individually. Any one of these modifications might affect the transitions at two adjacent locations; therefore, three test bits are involved in selecting the most appropriate modification. Consequently, the test data blocks to be analyzed consist of three consecutive bits of every test vector; two consecutive blocks overlap in exactly one bit position. Test vectors consisting of $n$ bits necessitate $\lceil(n-1) / 2\rceil$ blocks, consequently.

As the test vector bits earlier inserted to the scan chain pass through a larger number of scan cells, test vector transitions have weighted impacts on scan chain rippling depending on the transition position; the transitions between the bits with lower numbered indices toggle a larger number of scan cells. The asymmetry in the impact on the scan chain transitions stems from the fact that the test vectors applied to the circuit under test are overwritten by the test response bits without having a chance to shift out. The weighted impact of the test vector bits is taken into consideration in padding unspecified bits shared by adjacent blocks; in specifying such don't care bits, priority is given to the block containing bits with lower numbered indices. Section 4.3 dwells on this point in more detail.

\subsection{Analysis of fully specified test vectors}

A computationally efficient algorithm is employed wherein the test data is decomposed into blocks of three bits. For every block, the transition costs are calculated for each one of the eight transformations given in table 2. The modification with the minimal transition cost is implemented in the corresponding scan chain fragment.

Example 1: Figure 4 illustrates the modification of the scan chain based on the test set; in the first block, "010", and in the second block, " 110 ", occurs twice. Consequently, F2 and F7 are implemented in the corresponding parts of the scan chain; the number of scan cells toggled due to the direct application of the test vectors is 27 whereas the stimuli inserted to the modified scan chain result in 4 scan chain transitions.

\subsection{Analysis of partially specified test vectors}

If the given test set consists of test cubes, padding the unspecified bits and selecting the appropriate modification should be performed in an intertwined manner so as to obtain the minimum number of transitions. At any point, the proposed algorithm that examines the test data bits in chunks needs to tally the frequencies of 27 distinct combinations. The increase in the number of combinations to 27 stems from the necessity to consider unspecified data values as well as the two binary bit possibilities. Depending on which of the eight possible transformations given in table 1 is selected, a different padding may be suggested for the same set of input combinations; the appropriate padding needs to be stored in addition to the benefit information shown in table 2 on each column. For every block, the modification that yields the minimum number of transitions is selected for the corresponding scan chain fragment.

Unspecified bits at adjacent block overlaps may induce a sensitivity to the order in which test data blocks are handled. The block handled earlier determines the padding of the don't care bit in the intersection of two adjacent blocks. As the test data blocks that are shifted into the scan chain earlier pass through a larger number of scan cells, their weighted impact on scan chain rippling is expected to be larger; the blocks that contain the test data bits with lower numbered

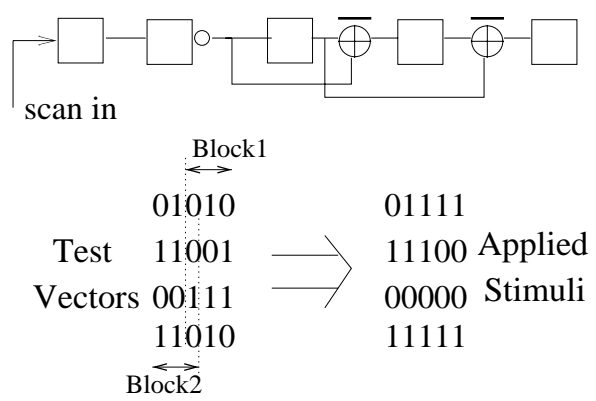

Figure 4. Test data and scan chain modification 


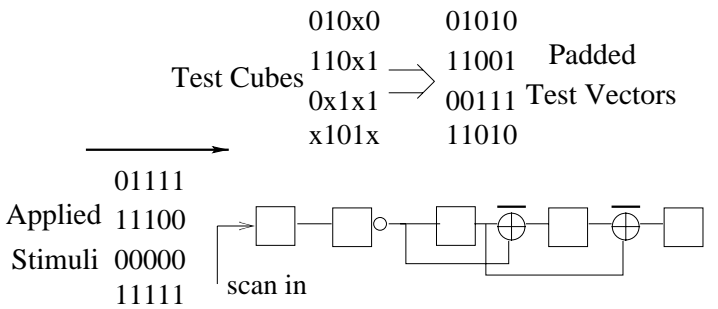

Figure 5. Test cube padding and modification of the scan chain

indices are handled first, consequently.

Example 2: The test set consisting of the test cubes $\{010 \times 0,110 \times 1,0 \times 1 \times 1, x 101 x\}$ can be padded as in Figure 5 to enable implementation of the modifications that will result in the minimum number, 4 , of scan chain transitions.

\section{Experimental Results}

The proposed test power reduction scheme has been applied to several combinational circuits in ISCAS85 [9] and fully-scanned circuits in ISCAS89 [10]. The test vectors and the test cubes that are used to compute the test power reductions achieved by the proposed methodology are generated by ATALANTA [11]. The proposed scheme is compared against another recent approach [5] that aims at reducing scan chain transitions. ${ }^{2}$

Table 3 demonstrates the results when the proposed modifications are implemented in the scan chain. The reduction in the total number of transitions in the scan chain, and the increase in area overhead are provided. Test power reductions for the test sets consisting of test cubes are computed by comparing the proposed scheme against the case wherein the test vectors are generated by randomly padding the unspecified bits of the test cubes. It should be noted that in evaluating our scheme we perform no vector dropping subsequent to the specification of don't care bits while such vector dropping is utilized in the case of random padding, thus understating somewhat the magnitude of the expected benefits of our technique. A noteworthy result is that the analysis of the test cubes of $c 6288$ offers almost no test power reduction; the low density of the unspecified bits in the test cubes along with the nearly uniform distribution of test data in the blocks limits the effectiveness of the proposed scheme. All the remaining cases display significant improvements; for the circuits $c 2670$ and $s 15850$, test power reductions of almost an order of magnitude are achieved. The higher comparative area reductions in the case of test cubes is most probably statistically insignificant.

\footnotetext{
${ }^{2}$ Other schemes, such as [6, 7], which aim at reducing the switching activity in the scan chain, constitute orthogonal approaches that can be applied in conjunction with the methodology we propose with no impact on the benefits provided by either; detailed comparisons are neither necessitated nor provided, consequently.
}

\begin{tabular}{|r|r|r|r|r|}
\hline \multirow{2}{*}{ Circuit } & \multicolumn{2}{|c|}{ Test Vectors } & \multicolumn{2}{c|}{ Test Cubes } \\
\cline { 2 - 5 } & Power(\%) & Area(\%) & Power(\%) & Area(\%) \\
\hline c2670 & 10.91 & 6.97 & 89.58 & 5.33 \\
\hline c3540 & 7.61 & 1.52 & 42.79 & 1.19 \\
\hline c5315 & 10.74 & 3.10 & 82.76 & 2.76 \\
\hline c6288 & 4.88 & 0.38 & 1.47 & 0.77 \\
\hline c7552 & 8.10 & 1.95 & 72.44 & 2.44 \\
\hline s713 & 16.44 & 5.33 & 74.62 & 4.67 \\
\hline s953 & 11.76 & 2.33 & 71.62 & 1.67 \\
\hline s1423 & 11.27 & 5.26 & 77.45 & 4.40 \\
\hline s5378 & 11.40 & 3.78 & 80.09 & 2.97 \\
\hline s9234 & 7.98 & 1.82 & 86.00 & 1.78 \\
\hline s15850 & 8.31 & 3.92 & 89.96 & 2.80 \\
\hline s35932 & 12.24 & 3.29 & 68.66 & 1.92 \\
\hline
\end{tabular}

Table 3. Test power reduction using inverters, XORs and XNORs

Even though padding for power minimization reduces scan chain transitions, slight test application time increases may result in comparison to the random padding case, particularly since no vector dropping has been effected in evaluating our technique. Table 4 demonstrates this tradeoff between test power and test application time; test power ratios of test vectors to those of test cubes are provided in the second column whereas the third column lists the test application time ratios of test cubes to those of test vectors. For instance, randomly padding the test cubes of the circuit $s 9234$ results in 216 test vectors; the proposed methodology for analyzing these 216 fully specified vectors achieves a reduction in transitions from 2.99 down to 2.75 million. Application of the proposed methodology directly to the test cubes, on the other hand, results in 237 test vectors with 0.42 million transitions. These, and analogous results summarized in table 4 , confirm the benefit of performing padding and scan chain modification in an intertwined manner, rather than randomly padding the test cubes and subsequently modifying the scan chain, despite the slight increase in test time.

Restricting the logic functions to be implemented in the

\begin{tabular}{|r|c|c|}
\hline Circuit & Power ratios & TAT multiples \\
\hline $\mathrm{c} 2670$ & 8.51 & 1.27 \\
\hline $\mathrm{c} 3540$ & 1.62 & 1.46 \\
\hline $\mathrm{c} 5315$ & 5.18 & 1.56 \\
\hline $\mathrm{c} 6288$ & 0.97 & 1.55 \\
\hline $\mathrm{c} 7552$ & 3.34 & 1.53 \\
\hline $\mathrm{s} 713$ & 3.29 & 1.10 \\
\hline $\mathrm{s} 953$ & 3.11 & 1.01 \\
\hline $\mathrm{s} 1423$ & 3.93 & 1.27 \\
\hline $\mathrm{s} 5378$ & 4.45 & 1.17 \\
\hline $\mathrm{s} 9234$ & 6.57 & 1.10 \\
\hline $\mathrm{s} 15850$ & 9.13 & 1.19 \\
\hline $\mathrm{s} 35932$ & 2.80 & 1.14 \\
\hline
\end{tabular}

Table 4. Tradeoff between test power and test application time 


\begin{tabular}{|r|r|r|}
\hline \multirow{2}{*}{ Circuit } & \multicolumn{2}{|c|}{ Power(\%) } \\
\cline { 2 - 3 } & Test Vectors & Test Cubes \\
\hline $\mathrm{c} 2670$ & 7.31 & 88.98 \\
\hline $\mathrm{c} 3540$ & 4.58 & 38.11 \\
\hline $\mathrm{c} 5315$ & 7.76 & 80.97 \\
\hline $\mathrm{c} 6288$ & 0.00 & 0.45 \\
\hline $\mathrm{c} 7552$ & 4.88 & 70.60 \\
\hline $\mathrm{s} 713$ & 12.20 & 72.02 \\
\hline $\mathrm{s} 953$ & 9.18 & 69.31 \\
\hline $\mathrm{s} 1423$ & 7.52 & 75.40 \\
\hline $\mathrm{s} 5378$ & 8.56 & 79.02 \\
\hline $\mathrm{s} 9234$ & 5.09 & 84.46 \\
\hline $\mathrm{s} 15850$ & 5.79 & 89.22 \\
\hline $\mathrm{s} 35932$ & 8.00 & 67.33 \\
\hline
\end{tabular}

Table 5. Test power reduction with no area overhead: Using inverters only

scan chain to inverters only fully eliminates any area cost; instead of inserting an inverter between two scan cells, the inverted output of the first scan cell can be connected to the input of the second one, thus necessitating no additional hardware. Table 5 shows the test power reductions thus achieved with no area overhead.

Table 6 demonstrates the improvements over the approach in [5] wherein the test cubes are padded appropriately for test power reduction with no modification in the scan chain. The number of transitions in the scan chain of the circuit $s 9234$ is 419,251 , when the scan chain is modified by the methodology we propose; an improvement of $25.79 \%$ is achieved over [5], wherein the number of transitions is 565,816 . These results further confirm the appreciable benefits of modifying the scan chain and padding the unspecified test bits in an intertwined manner, rather than solely padding the test cubes so as to reduce the transitions.

\section{Conclusion}

To enable parallelism in testing SOC cores, the average and peak test power associated with the cores should be reduced. The proposed methodology achieves this reduction by slightly modifying the scan chain based on the analysis of the given test set. A computationally efficient analysis of individual test data blocks for finding the optimal modification for the corresponding part of the scan chain is performed; the consequent algorithm leads to a global optimum, yet at linear run-times, as only a single pass over the test set is necessitated.

The proposed methodology achieves test power reductions through insertion of logic gates between the scan cells. Since no gate delays are introduced to the critical paths, no interference with the normal operation of the circuit ensues.

To demonstrate the efficacy of the proposed approach, we have applied it on several ISCAS85 and ISCAS89 benchmark circuits. The experimental results indicate that signif-

\begin{tabular}{|r|r|}
\hline Circuit & Power(\%) \\
\hline c2670 & 32.67 \\
\hline c3540 & 13.67 \\
\hline c5315 & 16.72 \\
\hline c6288 & 1.03 \\
\hline c7552 & 19.95 \\
\hline s713 & 31.74 \\
\hline s953 & 17.21 \\
\hline s1423 & 29.03 \\
\hline s5378 & 24.44 \\
\hline s9234 & 25.79 \\
\hline s15850 & 28.47 \\
\hline s35932 & 10.98 \\
\hline
\end{tabular}

\section{Table 6. Test power improvements over [5]}

icant test power reductions are achieved even with no area overheads; even higher power reductions can be achieved if slight additional area is expended in scan chain modifications.

\section{References}

[1] H. J. Wunderlich and S. Gerstendorfer, "Minimized power consumption for scan based BIST", in ITC, pp. 85-94, 1999.

[2] S. Wang and S. K. Gupta, "ATPG for heat dissipation minimization during test application", in ITC, pp. 250-258, 1998.

[3] T. Huang and K. Lee, "An input control technique for power reduction in scan circuits during test application", in ATS, pp. 315-320, 1999.

[4] N. Nicolici, B. M. Al-Hashimi and A. C. Williams, "Minimisation of power dissipation during test application in full-scan circuits using primary input freezing", IEEE TCOMP, vol. 47, n. 2, pp. 256-262, 1998.

[5] R. Sankaralingam, R. R. Oruganti and N. A. Touba, "Adapting scan architectures for low power operation", in VTS, pp. 35-40, 2000 .

[6] L. Whetsel, "Adapting scan architectures for low power operation", in ITC, pp. 863-872, 2000.

[7] V. Dabholkar, S. Chakravarty, I. Pomeranz and S. M. Reddy, "Techniques for minimizing power dissipation in scan and combinational circuits during test application", IEEE TCAD, vol. 17, n. 12, pp. 1325-1333, 1998.

[8] R. M. Chou, K. K. Saluja and V. D. Agrawal, "Scheduling tests for VLSI systems under power constraints", IEEE TVLSI, vol. 5, n. 2, pp. 175-184, 1997.

[9] F. Brglez and H. Fujiwara, "A Neutral Netlist of 10 Combinational Benchmark Circuits and a Target Translator in Fortran", ISCAS, June 1985.

[10] F. Brglez, D. Bryan and K. Kozminski, "Combinational Profiles of Sequential Benchmark Circuits", ISCAS, vol. 14, n. 2, pp. 1929-1934, May 1989.

[11] H. K. Lee and D. S. Ha, On the Generation of Test Patterns for Combinational Circuits, Technical Report 12-93, Department of Electrical Eng., Virginia Polytechnic Institute and State University. 